\title{
A Comparison of Three Fingerstick, Whole Blood Antibody Tests for Helicobacter pylori Infection: A United States, Multicenter Trial
}

W. D. Chey, M.D., F.A.C.G., U. Murthy, M.D., S. Shaw, M.D., A. Zawadski, J. Montague,

W. Linscheer, M.D., and L. Laine, M.D.

Divisions of Gastroenterology, University of Michigan Medical Center, Ann Arbor, Michigan; Veteran's Administration Medical Center, Syracuse, New York; University of Southern California Medical School, Los Angeles, California

OBJECTIVE: We compared three whole blood antibody tests for Helicobacter pylori (H. pylori) in a United States, multicenter trial.

METHODS: Patients referred for EGD at three medical centers were recruited. During EGD, biopsies were taken for histology and rapid urease testing (RUT). Immediately after endoscopy, patients underwent the antibody tests (FlexPack HP, Abbott Diagnostics; QuikVue, Quidel Corporation; AccuMeter, ChemTrak) using whole blood obtained by two to three fingersticks. Performance characteristics were calculated for each antibody test using the biopsy-based methods as a gold standard.

RESULTS: A total of 131 patients participated; 50 (38\%) patients had histological evidence of $H$. pylori infection. Using histology as a gold standard, the sensitivities of FlexPack HP, QuikVue, and Accumeter were 76\%, 78\%, and $84 \%$, respectively. Specificity was $79 \%$ with FlexPack HP and $90 \%$ with QuikVue and Accumeter. There were no significant differences in the performance of the three antibody tests though there was a trend toward superior performance for AccuMeter compared to FlexPack HP ( $p=$ 0.019). However, RUT proved superior to FlexPack HP using histology as a gold standard $(p=0.008)$. Using either concordant histology and RUT results or a positive histology or RUT to define active H. pylori infection, there was no statistically significant difference between the antibody tests.

CONCLUSIONS: There were no statistically significant differences in the performance of the three antibody tests. These tests proved only marginally sensitive in detecting patients infected with $H$. pylori. Clinicians should be aware of the limitations of these tests, particularly when using them as a sole means of testing for $H$. pylori. (Am $\mathbf{J}$ Gastroenterol 1999;94:1512-1516. (C) 1999 by Am. Coll. of Gastroenterology)

\section{INTRODUCTION}

Currently available diagnostic tests for Helicobacter pylori (H. pylori) infection include those requiring upper endoscopy with biopsy (for histology or rapid urease testing), and nonendoscopic techniques such as urea breath testing and serology. Currently, there is no true gold standard for the diagnosis of $H$. pylori infection. Most studies report sensitivities and specificities for histology, rapid urease testing (RUT), and urea breath testing of $>90 \%(1,2)$. Performance characteristics have been more variable for the serological tests (3). Serology is currently considered by many to be the most cost-effective initial diagnostic test for HP infection (4).

Both quantitative and qualitative antibody tests are commercially available. Quantitative serologic assays (ELISA) accurately identify infected patients who have not been previously treated for H. pylori (5). Disadvantages of quantitative serology testing include expense, need for specially trained personnel, and delay between the time the test is done and when results become available. More recently, a number of relatively inexpensive, simple to perform, officebased, qualitative antibody tests for $H$. pylori have become available. These qualitative tests can detect specific IgG antibodies to $H$. pylori using serum or whole blood. Serumbased qualitative tests have compared favorably in performance to the quantitative serological tests $(6,7)$, but require phlebotomy and centrifugation of blood samples. The recently introduced whole blood antibody tests, which use blood obtained by a fingerstick, offer all of the advantages of serum-based testing without the need for centrifugation of blood samples. The low cost, ease of performance, and rapid results of the whole blood tests make them ideal for the office-based practitioner, particularly in light of recent recommendations endorsing a "test and treat" strategy for young dyspeptic patients without alarm symptoms (8). However, the reported performance characteristics of the available whole blood antibody tests has varied considerably in the medical literature (9-15).

The aim of our study was to determine and compare the 
Table 1. Performance Characteristics and 95\% Confidence Intervals of the Antibody Tests and RUT Using Histology as a Gold Standard ( $\mathrm{n}=131$ Patients)

\begin{tabular}{lccccc}
\hline & Sensitivity & Specificity & PPV & NPV & Accuracy \\
\hline FlexPack & $38 / 50(76 \%)$ & $64 / 81(79 \%)$ & $38 / 55(69 \%)$ & $64 / 76(84 \%)$ & $(74-92 \%)$ \\
& $(62-87 \%)$ & $(69-87 \%)$ & $(55-81 \%)$ & $73 / 131(78 \%)$ \\
QuikVue & $39 / 50(78 \%)$ & $73 / 81(90 \%)$ & $39 / 47(83 \%)$ & $73 / 84(87 \%)$ & $112 / 131(85 \%)$ \\
& $(64-88 \%)$ & $(81-96 \%)$ & $(69-92 \%)$ & $(78-93 \%)$ & $(79-91 \%)$ \\
AccuMeter & $42 / 50(84 \%)$ & $73 / 81(90 \%)$ & $42 / 50(84 \%)$ & $73 / 81(90 \%)$ & $115 / 131(88 \%)$ \\
& $(71-93 \%)$ & $(81-96 \%)$ & $(71-93 \%)$ & $(81-96 \%)$ & $(82-94 \%)$ \\
RUT & $44 / 50(88 \%)$ & $75 / 81(93 \%)$ & $44 / 50(88 \%)$ & $75 / 81(93 \%)$ & $119 / 131(91 \%)$ \\
& $(76-95 \%)$ & $(85-97 \%)$ & $(76-95 \%)$ & $(85-97 \%)$ & $(86-96 \%)$ \\
\hline
\end{tabular}

$\mathrm{PPV}=$ positive predictive value; $\mathrm{NPV}=$ negative predictive value.

performance characteristics of three whole blood antibody tests for $H$. pylori using biopsy-based diagnostic methods as a gold standard in a United States, multicenter trial.

\section{MATERIALS AND METHODS}

\section{Patient Population}

Patients referred for upper endoscopy at three geographically diverse US medical centers were asked to participate in the study. Patients had to be $>18 \mathrm{yr}$ of age and able to understand and to give written informed consent. Patients were excluded if they had received treatment for $H$. pylori within $1 \mathrm{yr}$ of EGD, antibiotics or bismuth-containing compounds within 1 month of EGD, or a proton pump inhibitor within 7 days of EGD. This protocol was approved by the Institutional Review Boards of the participating centers.

\section{Study Protocol}

During endoscopy, biopsies were taken from the body $(>2)$ and antrum $(>2)$ of the stomach for histology (hematoxylin and eosin in all patients, and Giemsa in patients with histological gastritis but no $H$. pylori organisms by hematoxylin and eosin staining) and rapid urease testing (RUT; Pyloritek, Serim Research, Elkhart, IN, or Clotest, Trimed Specialties, Charlottesville, VA). Histology was performed by experienced gastrointestinal pathologists at each of the study sites.

Immediately after EGD, patients underwent three whole blood antibody tests: 1) FlexPack HP (Abbott Diagnostics, Abbott Park, IL); 2) Quick Vue (Quidel Corporation, San Diego, CA); and 3) AccuMeter (formerly known as the HpChek; ChemTrak, Sunnyvale, CA). In all three cases, antibody testing was performed using whole blood obtained with two to three fingersticks according to instructions provided by the manufacturer. The three antibody tests were performed in a nonrandomized fashion and interpreted by experienced operators who had no knowledge of the patient's endoscopic, RUT, or histological results.

\section{Interpretation of Data and Statistical Analysis}

Performance characteristics, including sensitivity, specificity, positive predictive value (PPV), negative predictive value (NPV), and accuracy were calculated for each whole blood antibody test using the biopsy-based diagnostic methods as the gold standard.

We first determined the performance characteristics of the antibody tests and RUT, using histology alone as a gold standard.

In our second analysis, active $H$. pylori infection was defined as a positive RUT and histology result. In this analysis, the presence of negative RUT and histology defined noninfected patients. Patients with discordant RUT and histology were excluded from this analysis. This type of analysis should present the "best case scenario" for the sensitivity and negative predictive value of the antibody tests.

In the final analysis, we defined active $H$. pylori infection as a positive RUT or histology result. We felt that this analysis was justified given the outstanding ( $>95 \%)$ specificity of both RUT and histology. In addition, this analysis most closely mimics how clinicians use RUT and histology in clinical practice. This analysis should present the "worst case scenario" for the sensitivity and negative predictive value of the antibody tests.

Ninety-five percent confidence intervals were calculated for the performance characteristics of the RUT and antibody tests (Tables 1-3). We compared the performance of the different tests using McNemar's test. The objective of this statistical analysis was to determine whether one test agreed with the gold standard more often than another. For this analysis, results from a pair of tests were summarized in a $2 \times 2$ table as agreeing or disagreeing with the gold standard. The two off-diagonal cells were then compared for statistically significant differences (agree for test A and disagree for test $\mathrm{B}$, versus agree for test $\mathrm{B}$ and disagree for test A). A $p<0.017$ (0.05/3 comparisons) defined a statistically significant difference between tests.

\section{RESULTS}

\section{Patient Population}

A total of 131 patients (54 women; mean age $54 \pm 2 \mathrm{yr}$, range 19-87 yr) participated in the study. The study population consisted of $63 \%$ Causcasians, $15 \%$ African-Americans, $16 \%$ Hispanic-Americans, and 6\% Asian-Americans. 
Table 2. Performance Characteristics and $95 \%$ Confidence Intervals of the Antibody Tests Using Concordant Histology and RUT Results as a Gold Standard $(\mathrm{n}=119)$

\begin{tabular}{lccccc}
\hline & Sensitivity & Specificity & PPV & NPV & Accuracy \\
\hline FlexPack & $34 / 44(77 \%)$ & $60 / 75(80 \%)$ & $34 / 49(69 \%)$ & $60 / 70(86 \%)$ & $(75-93 \%)$ \\
QuikVue & $(62-89 \%)$ & $(69-88 \%)$ & $(55-82 \%)$ & $(78-90 \%)$ \\
& $36 / 44(82 \%)$ & $68 / 75(91 \%)$ & $36 / 43(84 \%)$ & $68 / 76(89 \%)$ & $104 / 119(87 \%)$ \\
AccuMeter & $(67-92 \%)$ & $(82-96 \%)$ & $(69-93 \%)$ & $(81-95 \%)$ & $(81-93 \%)$ \\
& $39 / 44(89 \%)$ & $69 / 75(92 \%)$ & $39 / 45(87 \%)$ & $69 / 74(93 \%)$ & $108 / 119(91 \%)$ \\
& $(75-96 \%)$ & $(83-97 \%)$ & $(73-95 \%)$ & $(85-98 \%)$ & $(86-96 \%)$ \\
\hline
\end{tabular}

$\mathrm{PPV}=$ positive predictive value; NPV = negative predictive value.

After a retrospective review of our records, no participants had been previously treated for $H$. pylori. Eighteen percent of patients were found to have peptic ulcer disease at the time of endoscopy. Operators reported that all three antibody tests were easy to perform and to interpret.

\section{Test Characteristics}

Fifty (38\%) patients had histologic evidence of $H$. pylori infection. Relative to histology, sensitivities of the three fingerstick antibody tests were $76 \%, 78 \%$, and $84 \%$ for FlexPack HP, QuickVue, and the AccuMeter, respectively (Table 1). Specificity was 79\% with Flex Pak HP and 90\% for both QuickVue and the AccuMeter. Overall accuracy ranged from $78 \%$ with FlexPack HP to $88 \%$ with the Accumeter. Using McNemar's test, performance of the three antibody tests were not statistically significantly different though a trend toward superior performance of Accumeter compared to Flex Pack HP was noted $(p=0.019)$. Performance characteristics were also calculated for the RUT. Results of the RUT agreed with histology significantly more commonly than Flex Pack HP $(p=0.008)$. The performance of both QuickVue and the Accumeter were not statistically significantly different from the RUT.

When concordant histology and RUT results were used as a gold standard (H. pylori) infection defined as a positive histology and RUT), 44 (37\%) patients were found to be infected. Twelve patients (9\%) with discordant results on histology and RUT were excluded from this analysis. There were four patients with discordant histology and RUT results at each study site. Six patients had a positive RUT and negative histology and the remaining six had a negative RUT and positive histology. Using this gold standard, sensitivities for the antibody tests were 77\% for FlexPack HP, $82 \%$ for QuikVue, and $89 \%$ for the AccuMeter (Table 2). Specificity ranged from a low of $80 \%$ with FlexPack HP to
91\% and 92\% with QuikVue and the Accumeter, respectively. A full accounting of the performance characteristics using this gold standard is provided in Table 2.

When active infection was defined as a positive histology or RUT result, 56 (43\%) patients were found to be infected with $H$. pylori. The 12 patients with discordant histology and RUT results were included in this analysis. As expected, sensitivities of the antibody tests decreased using this gold standard (73\% for FlexPack HP, 71\% for QuikVue, and $78 \%$ for the AccuMeter). Specificities were $81 \%$ for FlexPack HP, 91\% for QuikVue, and 92\% with the AccuMeter (Table 3). Based on McNemar's test, there was a trend toward greater agreement with the gold standard for the Accumeter when compared with FlexPack HP $(p=0.021)$.

\section{DISCUSSION}

A recent technical review from the American Gastroenterological Association recommended that young patients with uncomplicated dyspepsia undergo testing for $H$. pylori and receive treatment if infected (8). Such a strategy would be most effectively carried out with a rapid, easy to perform, inexpensive test for $H$. pylori. Such an office-based test would allow the clinician to decide upon the need for $H$. pylori therapy in a single visit, thus obviating the need for follow-up phone management or a return visit to discuss test results and/or to initiate therapy. The rapid, qualitative, office-based antibody tests for $H$. pylori seem well suited to such a "test and treat" strategy. The first generation qualitative antibody assays required the use of serum, and thus the inconvenience of phlebotomy and centrifugation of blood samples. The newer, CLIA-waived whole blood fingerstick tests, however, are inexpensive and are considerably easier and less time consuming to perform. Although

Table 3. Performance Characteristics and $95 \%$ Confidence Intervals of the Antibody Tests Using Positive Histology or RUT to Define Active Infection $(\mathrm{n}=131)$

\begin{tabular}{lccccc}
\hline & Sensitivity & Specificity & PPV & NPV & Accuracy \\
\hline FlexPack & $41 / 56(73 \%)$ & $61 / 75(81 \%)$ & $41 / 55(75 \%)$ & $61 / 76(80 \%)$ & $(70-89 \%)$ \\
QuikVue & $(60-84 \%)$ & $(71-89 \%)$ & $(61-85 \%)$ & $(71-85 \%)$ \\
& $40 / 56(71 \%)$ & $68 / 75(91 \%)$ & $40 / 47(85 \%)$ & $68 / 84(81 \%)$ & $108 / 131(82 \%)$ \\
AccuMeter & $(58-83 \%)$ & $(82-96 \%)$ & $(72-94 \%)$ & $(71-89 \%)$ & $(75-89 \%)$ \\
& $44 / 56(79 \%)$ & $69 / 75(92 \%)$ & $44 / 50(88 \%)$ & $69 / 81(85 \%)$ & $113 / 131(86 \%)$ \\
& $(66-88 \%)$ & $(83-97 \%)$ & $(76-95 \%)$ & $(76-92 \%)$ & $(80-92 \%)$ \\
\hline
\end{tabular}

$\mathrm{PPV}=$ positive predictive value; $\mathrm{NPV}=$ negative predictive value. 
the whole blood antibody tests offer a potentially attractive alternative to routine serology or serum-based qualitative testing, most of the data addressing their performance characteristics has appeared only in abstract form (11-15). The available studies have reported variable performance characteristics for the whole blood antibody tests with sensitivity of $70-92 \%$ and specificity of $69-95 \%(9-15)$.

We report the performance characteristics of three whole blood antibody tests for $H$. pylori. Performance characteristics of FlexPack HP and the AccuMeter in the current study were comparable to those reported previously $(9,12)$. Using histology as a gold standard, there was not a statistically significant difference in the performance characteristics of the three antibody tests. However, FlexPack HP agreed significantly less often with a histological gold standard than the RUT $(p=0.008)$. Using a gold standard of either concordant histology and RUT or a positive histology or RUT to define active $H$. pylori infection, there was no statistically significant difference among the three antibody tests. However, using either a positive histology alone ( $p=$ $0.019)$ or positive histology or RUT $(p=0.021)$ to define active $H$. pylori infection, there did appear to be a trend toward superior performance for the AccuMeter when compared with FlexPack HP.

All three of the antibody tests evaluated use the principle of immunochromatography to detect $\operatorname{IgG}$ antibodies to $H$. pylori in whole blood, but they differ slightly in their specific test components. QuickVue and the AccuMeter contain immobilized purified $H$. pylori antigen in their test lines, but use different conjugates to detect patient antibodies to $H$. pylori. QuickVue uses labeled monoclonal anti-human IgG antibodies, whereas AccuMeter uses labeled "Protein G", which is a protein conjugate that binds IgG antibodies to $H$. pylori. The FlexPack HP test contains immobilized antihuman IgG, while using labeled $H$. pylori antigen as its conjugate. It is unclear whether these differences affect their performance characteristics, or whether other factors such as the use of different $H$. pylori antigens (which may vary between strains of the organism), differences in the test kit microenvironments (which may affect ligand binding kinetics), or regional differences in pretest likelihood of infection are important.

Interestingly, performance characteristics of the three antibody tests did vary among the different study sites. Unfortunately, the relatively small numbers of patients at the individual sites precluded a detailed statistical comparison of the antibody tests. For this reason, these data have not been included in this article. The pretest probability of $H$. pylori infection was at least partially responsible for the regional variation in the positive and negative predictive values. A more provocative explanation for the differences in sensitivity and specificity observed between study sites is regional antigenic variability in $H$. pylori strains (16). If this is the case, local validation of antibody test performance characteristics may be necessary.

A limitation of our study protocol was the potential in- clusion of patients tested for $H$. pylori $>1$ yr before upper endoscopy. We have reviewed the records of the 131 patients enrolled in this study and no patients had been previously treated for $H$. pylori. Even if a small number of previously treated patients had been enrolled, their effect on the results would be minimal. In support of this statement, we offer two arguments. First, studies have suggested that between $35 \%$ and $94 \%$ of those successfully cured of $H$. pylori will experience seroconversion at $1 \mathrm{yr}(17,18)$. One could argue that the percentage of patients expected to have a negative whole blood antibody test 1 year after $H$. pylori eradication could be even higher as the published studies have utilized very sensitive ELISAs. Consequently, only a fraction of previously treated patients would be expected to have a positive antibody test at the time of enrollment. Perhaps more importantly, the inclusion of previously treated patients would be expected to produce false positive antibody test results and, in turn, adversely affect the specificity of these tests. In fact, the specificities of two of the three whole blood antibody tests was $>90 \%$. We would stress that the current data do not support the use of the whole blood antibody tests as a method of establishing $H$. pylori eradication.

We feel that the performance characteristics yielded by the three whole blood antibody tests have important implications for the clinician. The ideal test for a "test and treat" strategy would be both sensitive and specific for $H$. pylori infection. However, faced with the choice of a more sensitive or specific test, we would argue in favor of the more sensitive test. As $H$. pylori infection is clearly associated with peptic ulcer disease $(19,20)$ and gastric malignancy (20) and perhaps associated with nonulcer dyspepsia (21), it is important to identify those individuals with infection. Certainly for those dyspeptic patients with peptic ulcer disease, the potential sequelae of undiagnosed infection, both in terms of morbidity and of the health care resource use associated with further evaluation and misguided therapies, could be quite substantial. As such, clinicians using whole blood antibody tests in the community should be aware of their marginal sensitivities.

In conclusion, when histology was used as a gold standard, RUT proved superior to Flex Pak HP. There were no statistically significant differences in the performance characteristics of the three whole blood antibody tests evaluated. The whole blood antibody tests yielded sensitivities of questionable acceptability. Clinicians should be aware of the performance characteristics and limitations of these tests, particularly when using them as a sole means of identifying patients infected with $H$. pylori.

\section{ACKNOWLEDGMENTS}

Part of this work was presented at Digestive Diseases Week (New Orleans, LA, May 1998). Whole blood antibody tests were provided by Abbott Diagnostics, Abbott Park, IL; 
Proctor and Gamble, Cincinnati, $\mathrm{OH}$; and ChemTrak, Sunnyvale, CA.

The authors acknowledge the invaluable contributions of A. Fayyed, R. Bandekar, J. Barnett, G.H. Elta, L. Fisher, W.L. Hasler, T. Nostrant, and J. Scheiman at the University of Michigan Medical Center.

Reprint requests and correspondence: William D. Chey, M.D., University of Michigan Medical Center, 3912 Taubman Center, Box 0362, Ann Arbor, MI 48109.

Received Aug. 13, 1998; accepted Feb. 23, 1999.

\section{REFERENCES}

1. Cutler AF, Havstad S, Ma CK, et al. Accuracy of invasive and noninvasive tests to diagnose Helicobacter pylori infection. Gastroenterology 1995;109:136-41.

2. Thijs JC, van Swet AA, Thijs WJ, et al. Diagnostic tests for Helicobacter pylori: A prospective evaluation of their accuracy, without selecting a single test as the gold standard. Am J Gastroenterol 1996;91:2125-9.

3. Mégraud F. Diagnosis and candidates for treatment of Helicobacter pylori infection. Gastroenterology 1997;113:S93-8.

4. Ofman JJ, Etchason J, Fullerton S, et al. Management strategies for Helicobacter pylori-seropositive patients with dyspepsia: Clinical and economic consequences. Ann Intern Med 1997;126:280-91.

5. Evans DJ, Evans DG, Graham DY, et al. A sensitive and specific serologic test for detection of campylobacter pylori infection. Gastroenterology 1989;96:1004-8.

6. Chey WD, Murthy UK, Linscheer WG, et al. A comparison between 4 different commercially available serology tests for Helicobacter pylori. Gastroenterology 1996;110:A80 (abstract).

7. Graham DY, Evans DJ, Peacock J, et al. Comparison of rapid serological tests (Flexsure HP and Quickvue) with conventional ELISA for detection of Helicobacter pylori infection. Am J Gastroenterol 1996;91:942-8.

8. Talley NJ, Silverstein MD, Agreus L, et al. AGA technical review: Evaluation of dyspepsia. Gastroenterology 1998;114: 582-95.

9. Chey WD, Murthy UK, Linscheer W, et al. The Chemtrak HP Chek fingerstick whole blood serology test for the detection of
Helicobacter pylori infection. Am J Gastroenterol 1998;93: 16-19.

10. Borody TH, Andrews P, Shortis NP. Evaluation of whole blood antibody kit to detect active Helicobacter pylori infection. Am J Gastroenterol 1996;91:2509-12.

11. Lahaie RG, Ricard N. Validation of Helisal whole blood, serum, and saliva tests for the non-invasive diagnosis of $H$. pylori infection. Gastroenterology 1996;110:A167 (abstract).

12. Sadowski D, Cohen H, Laine L, et al. Evaluation of the Flexsure HP fingerstick blood test for the detection of $H$. pylori infection. Gastroenterology 1996;110:A246 (abstract).

13. Cohen H, Retama B, Johnson C, et al. Evaluation of a rapid test to detect $\operatorname{IgG}$ antibodies to Helicobacter pylori using fingerstick whole blood samples. Gastroenterology 1996;110: A83 (abstract).

14. Chey WD, Murthy U, Linscheer W, et al. A comparison of 2 commercially available fingerstick, whole blood antibody test for H. pylori infection. Gastroenterology 1998;114:A90 (abstract).

15. Faigel DO, Magaret N, Lieberman DA, Fennerty MB. Rapid whole blood antibody tests for the diagnosis of Helicobacter pylori $(\mathrm{Hp})$ infection: Comparison with histology, rapid urease, urea breath and serum based testing. Gastroenterology 1998;114:A117 (abstract).

16. Hook-Nikanne J, Perez-Perez GI, Blaser MJ. Antigenic characterization of Helicobacter pylori strains from different parts of the world. Clin Diag Lab Immunol 1997;4:592-7.

17. Cutler AF, Prasad VM. Long term follow-up of Helicobacter pylori serology after successful eradication. Am J Gasteroenterol 1996;91:85-8.

18. Wang WM, Chen CY, Jan CM, et al. Long term follow-up on serological study after triple therapy of Helicobacter pylori associated duodenal ulcer. Am J Gastroenterol 1994;89: 1793-6.

19. Van Der Hulst RWM, Rauws EAJ, Köycö B, et al. Prevention of ulcer recurrence after eradication of Helicobacter pylori: A prospective long-term follow-up study. Gastroenterology 1997;113:1082-6.

20. Graham DY. Helicobacter pylori infection in the pathogenesis of duodenal ulcer and gastric cancer: A model. Gastroenterology 1997;113:1983-91.

21. McCarthy C, Patchett S, Collins RM, et al. Long-term prospective study of Helicobacter pylori in nonulcer dyspepsia. Dig Dis Sci 1995;540:114-9. 